BMJ Open

Diabetes

Research

\& Care

\title{
Altered pain processing in patients with type 1 and 2 diabetes: systematic review and meta-analysis of pain detection thresholds and pain modulation mechanisms
}

\author{
Eva Sierra-Silvestre, ${ }^{1,2}$ Mari Somerville, ${ }^{1}$ Leanne Bisset, ${ }^{1,3}$ \\ Michel W Coppieters (i) 3,4
}

To cite: Sierra-Silvestre E, Somerville M, Bisset L, et al. Altered pain processing in patients with type 1 and 2 diabetes: systematic review and meta-analysis of pain detection thresholds and pain modulation mechanisms. BMJ Open Diab Res Care 2020;8:e001566. doi:10.1136/ bmjdrc-2020-001566

- Additional material is published online only. To view, please visit the journal online (http://dx.doi.org/10.1136/ bmjdrc-2020-001566).

Received 17 May 2020 Revised 24 June 2020 Accepted 6 July 2020
Check for updates

(c) Author(s) (or their employer(s)) 2020. Re-use permitted under CC BY-NC. No commercial re-use. See rights and permissions. Published by BMJ.

For numbered affiliations see end of article.

Correspondence to Professor Michel W Coppieters; m.coppieters@griffith.edu.au

\section{ABSTRACT}

The first signs of diabetic neuropathy typically result from small-diameter nerve fiber dysfunction. This review synthesized the evidence for small-diameter nerve fiber neuropathy measured via quantitative sensory testing (QST) in patients with diabetes with and without painful and non-painful neuropathies. Electronic databases were searched to identify studies in patients with diabetes with at least one QST measure reflecting small-diameter nerve fiber function (thermal or electrical pain detection threshold, contact heat-evoked potentials, temporal summation or conditioned pain modulation). Four groups were compared: patients with diabetes (1) without neuropathy, (2) with non-painful diabetic neuropathy, (3) with painful diabetic neuropathy and (4) healthy individuals. Recommended methods were used for article identification, selection, risk of bias assessment, data extraction and analysis. For the meta-analyses, data were pooled using random-effect models. Twenty-seven studies with 2422 participants met selection criteria; 18 studies were included in the meta-analysis. Patients with diabetes without symptoms of neuropathy already showed loss of nerve function for heat (standardized mean difference (SMD): 0.52, $p<0.001$ ), cold (SMD: $-0.71, p=0.01$ ) and electrical pain thresholds (SMD: 1.26, $p=0.01$ ). Patients with non-painful neuropathy had greater loss of function in heat pain threshold (SMD: $0.75, p=0.01$ ) and electrical stimuli (SMD: 0.55, $p=0.03$ ) compared with patients with diabetes without neuropathy. Patients with painful diabetic neuropathy exhibited a greater loss of function in heat pain threshold (SMD: 0.55, $p=0.005$ ) compared with patients with non-painful diabetic neuropathy. Small-diameter nerve fiber function deteriorates progressively in patients with diabetes. Because the dysfunction is already present before symptoms occur, early detection is possible, which may assist in prevention and effective management of diabetic neuropathy.

\section{INTRODUCTION}

Diabetes affects $8.5 \%$ of the total adult population. ${ }^{1}$ Elevated sugar levels over time can produce tissue damage at different levels to the nervous system. Among the various forms of diabetic neuropathy, distal symmetrical polyneuropathy (DSPN) is the most common long-term diabetic complication, affecting up to $50 \%$ of patients with diabetes. ${ }^{2}$ Of those with DSPN, $13 \%$ report pain associated with the DSPN, ${ }^{3}$ which impacts on their daily activities and quality of life. DSPN is defined as a bilateral, length-dependent sensorimotor neuropathy. ${ }^{4}$ Typically, symptoms of DSPN are initially localized at the toes, before progressively affecting the feet and lower legs. DSPN in the hands is less common, and generally manifests itself after DSPN has developed in the lower limbs. Other forms of diabetic neuropathy may coexist, like cardiovascular autonomic neuropathy or atypical forms of diabetic neuropathy (eg, carpal tunnel syndrome).$^{5}$

DSPN affects multiple nerves and various nerve fiber types. ${ }^{4}$ Sensory nerve fibers are typically affected before motor nerve fibers. ${ }^{6}$ In the early stages, small-diameter sensory nerve fibers responsible for thermal, electrical and pain perception (ie, A $\delta$ and C-fibers) are affected. ${ }^{7}$ As DSPN progresses, large-diameter sensory nerve fibers responsible for touch and vibration perception (ie, $A \beta$ and $A \gamma$ fibers) are affected. ${ }^{8}$ This may manifest itself clinically as either loss of nerve function resulting in hypoalgesia (small-diameter nerve fibers) or hypoesthesia (large-diameter nerve fibers) or gain of function, resulting in pain (smalldiameter nerve fibers) or paraesthesia (largediameter nerve fibers). Motor neuropathy (A $\alpha$ fibers) may result in muscle weakness and may contribute to muscle cramps. ${ }^{8}$

Small fiber neuropathy can manifest as spontaneous and stimulus-evoked distal pain, deep aching and sensory loss. ${ }^{9}$ Patients with small fiber neuropathy are at increased 
risk for foot ulcerations and amputations. ${ }^{10}$ In the last 10-15 years, there is a growing interest in small fiber neuropathy in various pathologies, such as carpal tunnel syndrome $^{11}$ and complex regional pain syndrome. ${ }^{12}$ Similarly, various methods have been employed to assess small-diameter nerve pathology in diabetes. ${ }^{6}$ Available techniques include skin biopsy and corneal confocal microscopy to assess morphological changes. To evaluate function, quantitative sensory testing (QST), laserevoked potentials and sudomotor function can be assessed. Static QST measures are used to quantify gain and/or loss of somatosensory function in small-diameter and large-diameter nerve fibers. Dynamic QST measures are used to gain insights in altered pain processing in the central nervous system, such as facilitation (eg, temporal and spatial summations) and inhibition (eg, conditioned pain modulation).

As DSPN significantly increases the morbidity of diabetes ${ }^{13}$ early detection and management of small fiber neuropathy is of cardinal importance. Multiple studies have used QST to document small fiber neuropathies in patients with diabetes with and without DSPN (eg, see Raputova $\left.e t a l^{14-19}\right)$. As findings from individual studies are sometimes conflicting and unable to provide a comprehensive insight, a systematic review and metaanalysis is required. Therefore, the aim of this study was to review and summarize the evidence on altered pain thresholds and pain modulation in patients with diabetes without and with DSPN (both painful and non-painful).

\section{RESEARCH DESIGN AND METHODS}

This systematic review and meta-analysis is reported in accordance with the Preferred Reporting Items for Systematic Reviews and Meta-Analysis statement. ${ }^{20}$ The review has been registered in PROSPERO (CRD42018088173) and the protocol is available online. ${ }^{21}$

\section{Eligibility criteria}

Studies were considered for inclusion in this review if they met the following criteria: (1) cohort studies, casecontrol studies or clinical trials conducted in adults (ie, at least 18 years of age), diagnosed with diabetes (type 1 or 2), without or with DSPN (painful or non-painful), with or without a healthy pain-free control group; (2) the study included at least one outcome related to detecting pain thresholds and/or pain modulation; and (3) the study included at least one of the following comparisons: patients with diabetes without DSPN versus patients with non-painful DSPN; patients with non-painful DSPN versus patients with painful DSPN; or patients with diabetes with or without DSPN versus healthy controls.

\section{Outcome measures}

Pain thresholds for the sensory modalities of interest (ie, heat, cold, pressure, pain by means of electrical stimulation and contact heat-evoked potentials) and pain modulation measures (ie, temporal summation or conditioned pain modulation) were extracted. Only quantitative outcomes were considered. If different body locations where measured within the same study (eg, great toe and arch of the foot), the location most commonly used among all the studies was selected.

\section{Data sources and searches}

Combinations of controlled vocabulary, Medical Subject Headings (MeSH) and free-text terms were developed in collaboration with a university health liaison librarian. MEDLINE (via EBSCO), CINAHL (via EBSCO), Embase (via Elsevier), the Cochrane Library, SPORTDiscus, Web of Science and PEDro were searched from their respective inception dates to $16^{\text {th }}$ March 2019. Reference lists from the included papers were screened for additional potentially eligible studies. Studies published in a peerreviewed journal in any language or any type of publication status were considered. An example of the search string can be found in the protocol paper. ${ }^{21}$

\section{Study selection}

Records were imported to Covidence (Veritas Health Innovation, Australia) ${ }^{22}$ for screening. One investigator (ES-S) screened titles and abstracts, and two independent investigators (ES-S and MS) screened full-text records. When there was disagreement, the two investigators discussed the eligibility, and if the disagreement could not be resolved, a third investigator (LB) was consulted. Unweighted Cohen's kappa ( $\kappa)$ coefficient was calculated to quantify the agreement in full-text selection between the reviewers. Agreement was scored as fair $(0.40-0.59)$, good $(0.60-0.74)$ or excellent $(>0.75) .{ }^{23}$

\section{Data collection process}

The data were extracted by one investigator (ES-S). The accuracy of the data extraction was verified by a second investigator (MS) who independently extracted data from a randomly selected subset of papers $(20 \%$ of the total). The level of agreement for extracted mean, SD and sample size was calculated using a two-way random single measure intraclass correlation coefficient $\left(\mathrm{ICC}_{2,1}\right)$. Data were extracted from each paper for (1) manuscript information (author and year); (2) study design; (3) patient information (sample size, age, sex, body mass index, type of diabetes, years since diabetes diagnosis, presence and type of neuropathy, disability and quality of life; (4) pain thresholds (cold pain threshold, heat pain threshold, pressure pain threshold, pain threshold by means of electrical stimuation and contact heat-evoked potentials); and (5) pain modulation variables (temporal summation and conditioned pain modulation).

\section{Risk of bias in individual studies}

Two investigators (ES-S and MS) analyzed the risk of bias in each paper using the Downs and Black checklist. ${ }^{24}$ This checklist was developed for healthcare interventions to assess the methodological quality of randomized controlled trials and non-randomized studies. It assesses 27 items categorized into (1) reporting, (2) external validity, (3) internal validity-bias, (4) internal 
validity-confounding (selection bias), and (5) power. For the purpose of this systematic review, items 5, 9-12, $14,17,19,23,24,26$ and 27 were not considered as they address aspects related to longitudinal or interventional studies. The maximum final score of the abbreviated checklist was therefore 14. Discrepancies in quality rating between the investigators were resolved by discussion, and when necessary, a third investigator (LB) was consulted. Reliability for risk of bias was assessed using an ICC $_{2,1}$ with $95 \%$ CIs.

\section{Synthesis of results}

A quantitative synthesis (meta-analysis) was performed when possible. The results were pooled using a randomeffect meta-analysis when appropriate. The summary statistic calculated for each study was the standardized mean difference (SMD) with 95\% CI for each outcome of interest. When outcomes were reported in subgroups (eg, for diabetes type 1 and type 2), data were combined by calculating the weighted average.

Heterogeneity between studies was analyzed using $\mathrm{I}^{2}$ statistic. $\mathrm{I}^{2}$ values were interpreted according to the cutoff points of $25 \%$ (low heterogeneity), $50 \%$ (moderate heterogeneity) and $75 \%$ (high heterogeneity). ${ }^{23}$

Metaregression analysis was planned to examine whether group differences were influenced by risk of bias measured with the Downs and Black checklist. Studies with a score of $<7$ were considered to have low risk of bias, and studies with a score of $\geq 7$ were considered to have high risk of bias. A metaregression was only considered when at least 10 studies were available for analysis. ${ }^{23}$

Meta-analytical comparison of the different pain threshold between the subgroups of patients was performed using the 'metafor' package from the R-project V.2.0.0 (www.metafor-project.org). ${ }^{25}$ If a quantitative synthesis was not possible due to a lack of available data or authors failing to respond when additional information was required, a narrative synthesis was performed instead.

\section{RESULTS}

\section{Study characteristics}

Initial searches yielded 3841 unique records. After screening titles and abstracts and 359 full texts were reviewed, 27 papers were included in the qualitative synthesis and 18 in the meta-analysis (online supplementary figure 1). Agreement between the two raters regarding the study selection was excellent, with a Cohen $\kappa$ of $0.86(\mathrm{p}<0.001,95 \%$ CI 0.58 to 1.14$)$. Agreement on data extraction was excellent for all measures $\left(\mathrm{ICC}_{2,1} \geq 0.95,95 \% \mathrm{CI} 0.88\right.$ to 0.99$)$.

The included studies were published between 1987 and 16 March 2019, with $60 \%$ of the papers published in the past 10 years. Eight studies were cohort studies ${ }^{146-32}$ and 19 studies were cross-sectional studies. ${ }^{16-1933-47}$ Seven studies ${ }^{14} 31373841-43$ included a group of patients with painful DSPN (n=384); 23 studies $^{14} 16-19$ 26-35 $39-41$ 43-47 included a group of patients with non-painful DSPN $(\mathrm{n}=754) ; 13$ studies $^{18} 192930$ 33-37 44-47 included a group of patients with diabetes without DSPN $(\mathrm{n}=635)$; and 24 studies ${ }^{16-19} 26-2830-46$ included a group of healthy individuals $(\mathrm{n}=615)$. Nine studies included only participants with type 2 diabetes ${ }^{17-19} 343637414244$; 11 included both participants with type 1 and type 2 diabetes ${ }^{141626-2830-32394143}$; and 7 studies did not report the type of diabetes. ${ }^{29} 33353845-47$ Based on the studies that reported the type of diabetes, $17.6 \%$ were patients with type 1 diabetes. The participants from one study ${ }^{40}$ were not included due to a mismatch between the data reported in the text compared with the tables, and we were unable to receive clarification from the authors which data were correct.

The mean age, sex (\% women) and mean duration of diabetes for the different groups were painful DSPN: 57.8 (SD 10.9) years, 39.8\% women, 18.1 (SD 8.9) years; non-painful DSPN: 58.4 (SD 10.7) years, $34.5 \%$ women, 17.9 (SD 0.3) years; diabetes without DSPN: 49.7 (SD 9.2) years, $56.2 \%$ women, 9.4 (SD 4.8) years; and healthy: 48.1 (SD 12.2) years, 53.0\% women. Three studies 173246 reported results on health-related quality of life using an adapted version of the Neurological Symptoms Score, ${ }^{48}$ or the 36-Item Short Form Health Survey (SF-36). Patients with DSPN showed a lower health-related quality of life compared with patients with diabetes without DSPN and compared with healthy participants.

The criteria to diagnose diabetic neuropathy differed between studies. Eight studies ${ }^{1417283031424346}$ used confirmatory tests for neuropathy, ${ }^{6}$ such as intraepidermal nerve fiber density ${ }^{143142}$ or electrodiagnostic tests. ${ }^{14172830314346}$ Five studies included patients with possible or probable DSPN based on questionnaires such as the Leeds Assessment of Neuropathic Symptoms and Signs,${ }^{19}$ the Toronto Clinical Scoring system, ${ }^{18}$ the Michigan Neuropathy Screening Index, ${ }^{34}$ or the American Diabetes Association criteria. ${ }^{29} 32$ Other tests used were based on perception thresholds (eg, tuning fork), a combination of perception thresholds, ${ }^{16} 26273841$ or bedside neurological examination (eg, ankle reflexes and expert criteria) 33353945 (online supplementary table 1 ).

The pain detection thresholds measured were heat pain threshold (17 studies), ${ }_{14} 1819$ 28-31 36 37 39-41 43-47 cold pain threshold (9 studies), 141929313637404647 pressure pain threshold (5 studies), ${ }^{14} 16262731$ contact heat-evoked potentials (3 studies), ${ }^{1735} 42$ and pain threshold by means of electrical stimulation (2 studies). ${ }^{33} 34$ Two studies reported temporal summation ${ }^{32} 38$ and one study ${ }^{38}$ measured conditioned pain modulation (online supplementary table 1 ).

\section{Risk of bias within studies}

The mean Downs and Black score across studies was 9 , ranging from 6 to 11. The item least frequently listed related to reporting adverse events. Many studies failed to provide information about the recruitment of participants (period of time or whether all participants were recruited from the same population) or blinding of the 
Heat pain threshold

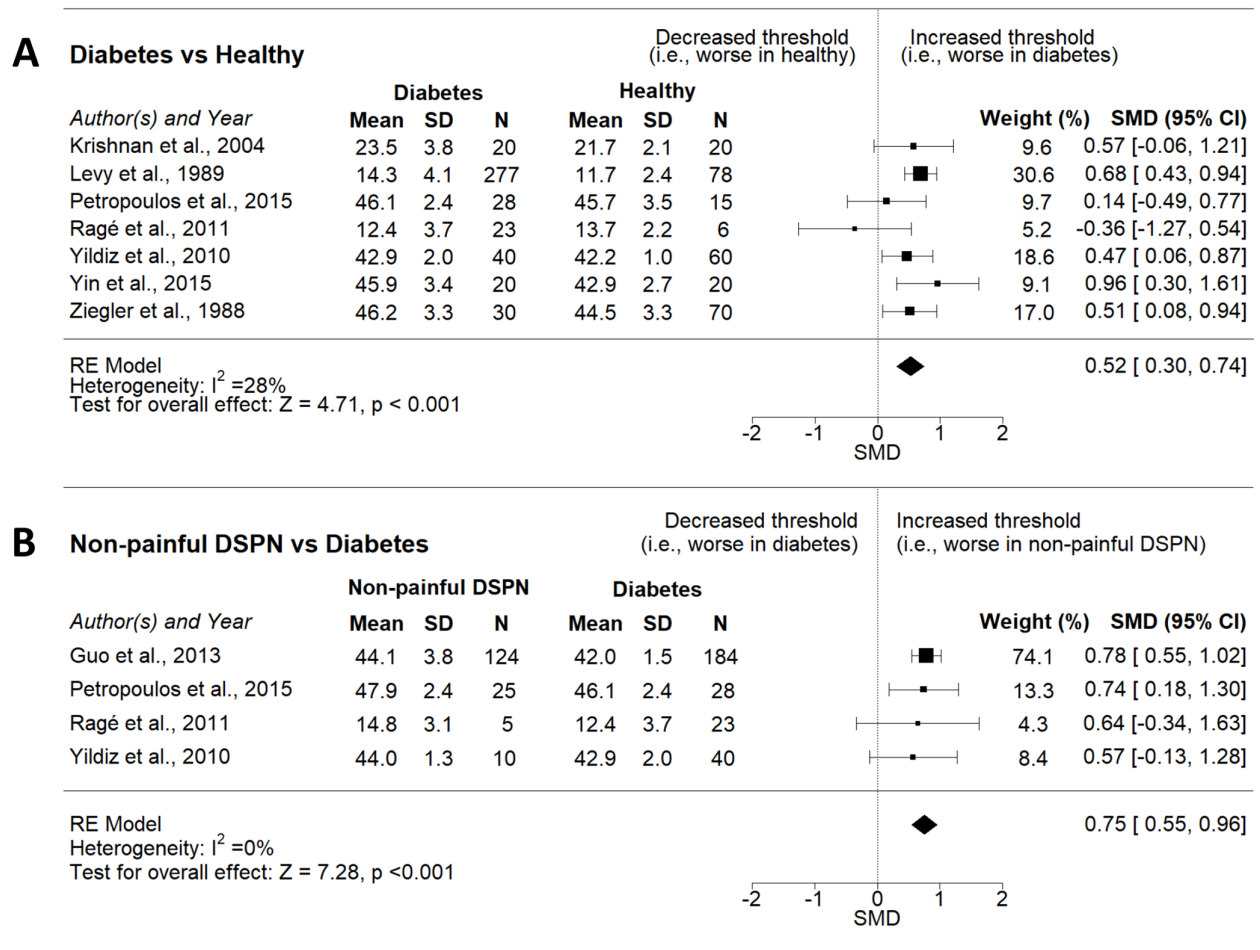

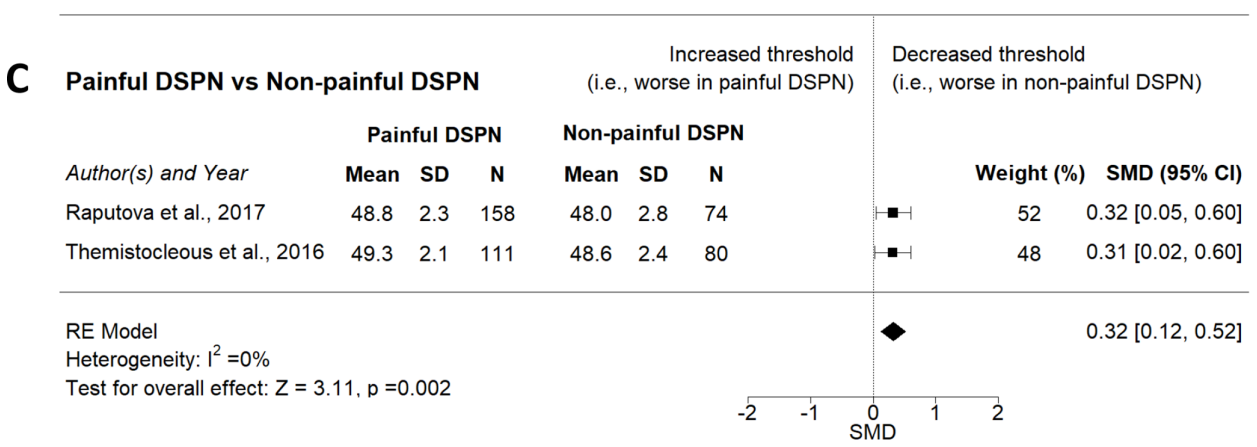

Figure 1 Forest plots for heat pain threshold for patients with painful DSPN versus non-painful DSPN, non-painful DSPN versus diabetes and diabetes versus healthy. DSPN, distal symmetrical polyneuropathy; RE, random effect; SMD, standardized mean difference.

measurements (online supplementary table 2). Agreement between the two raters for the Downs and Black scores was excellent, with a Cohen $\kappa$ of $0.82(\mathrm{p}<0.001$, $95 \%$ CI 0.71 to 0.93$)$.

\section{Synthesis of results \\ Heat pain threshold}

Pooled data from seven studies ${ }^{18} 193637$ 44-46 showed that patients with diabetes without DSPN $(n=438)$ had increased heat pain threshold (ie, pain at higher temperatures) compared with healthy individuals $(\mathrm{n}=269)$ (SMD: $0.52,95 \%$ CI 0.30 to $0.74 ; \mathrm{Z}=4.71, \mathrm{p}<0.001)$. The results from one study ${ }^{45}$ were presented separately for men and women, so we combined them into a single group prior to further evaluation. Four studies ${ }^{18} 192946$ with low heterogeneity $\left(\mathrm{I}^{2}=0 \%\right)$ demonstrated that patients with non-painful DSPN $(n=164)$ had increased heat pain threshold compared with patients with diabetes without DSPN ( $\mathrm{n}=275$ ) (SMD: $0.75,95 \%$ CI 0.55 to $0.96 ; \mathrm{Z}=7.28$, $\mathrm{p}<0.001$; figure 1 ). Two studies ${ }^{1431}$ of low heterogeneity
$\left(\mathrm{I}^{2}=0 \%\right)$ revealed that patients with painful DSPN $(n=269)$ had a decreased heat pain threshold (ie, detect pain at lower temperatures) compared with non-painful DSPN ( $\mathrm{n}=154$ ) (SMD: 0.32 , 95\% CI 0.12 to 0.52 ; $\mathrm{Z}=-3.11$, $\mathrm{p}=0.002$ ) (figure 1). Three studies ${ }^{18} 1946$ with moderate heterogeneity $\left(\mathrm{I}^{2}=60 \%\right)$ revealed that patients with nonpainful DSPN $(\mathrm{n}=40)$ presented with increased heat pain threshold compared with healthy controls $(n=81)$ (SMD 1.02, 95\% CI 0.28 to $1.78 ; \mathrm{Z}=2.63$, $\mathrm{p}=0.01$ ) (online supplementary figure 2). The findings of one study ${ }^{41}$ could not be pooled because the reported values could not be converted to degrees. This study showed higher values for heat pain onset in patients with non-painful DSPN versus painful DSPN. This difference was significant in the comparison between patients with nonpainful DSPN and healthy individuals. No difference was observed between patients with painful DSPN and healthy individuals. 
Cold pain threshold

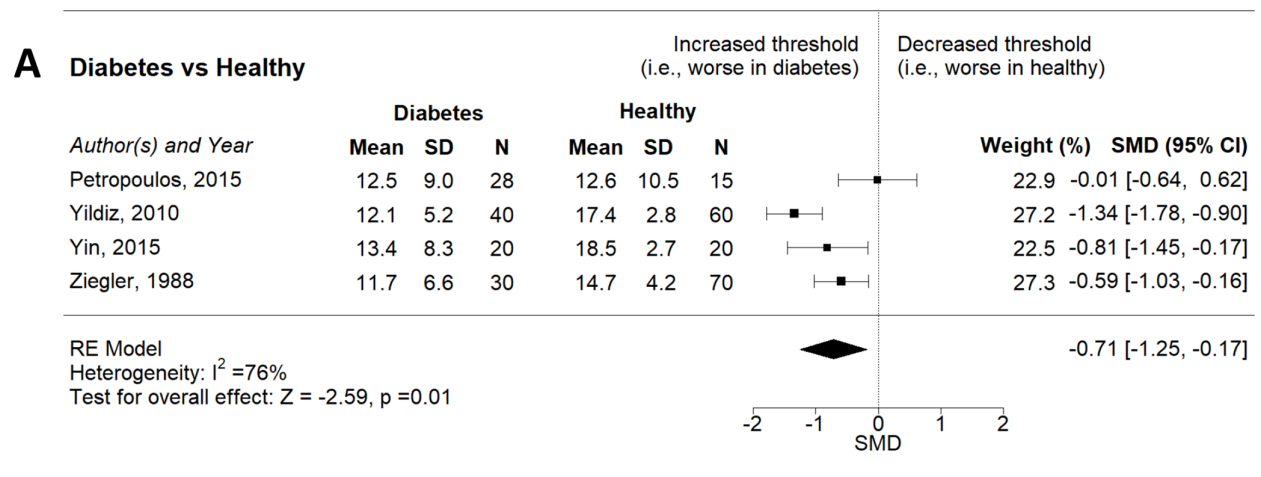

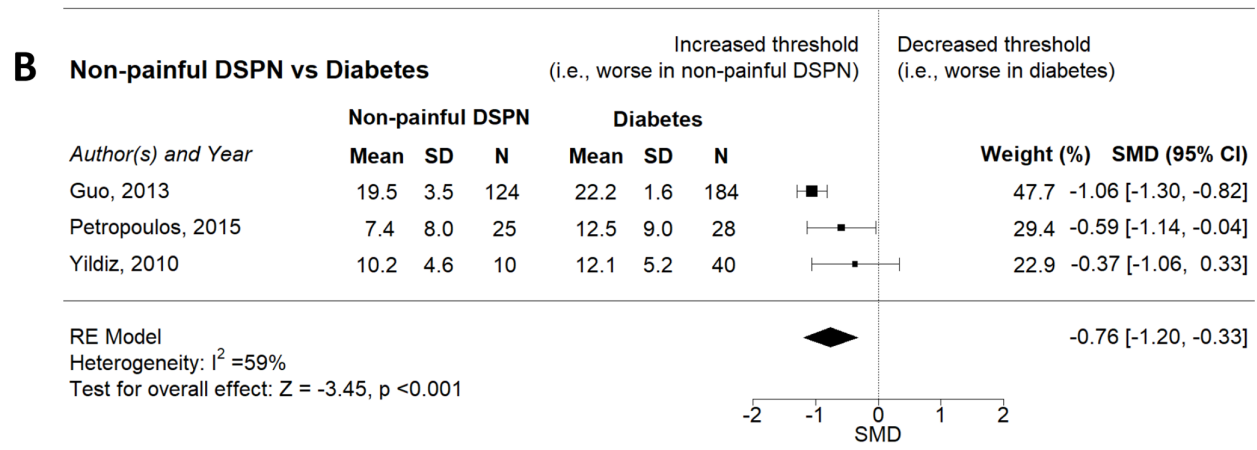

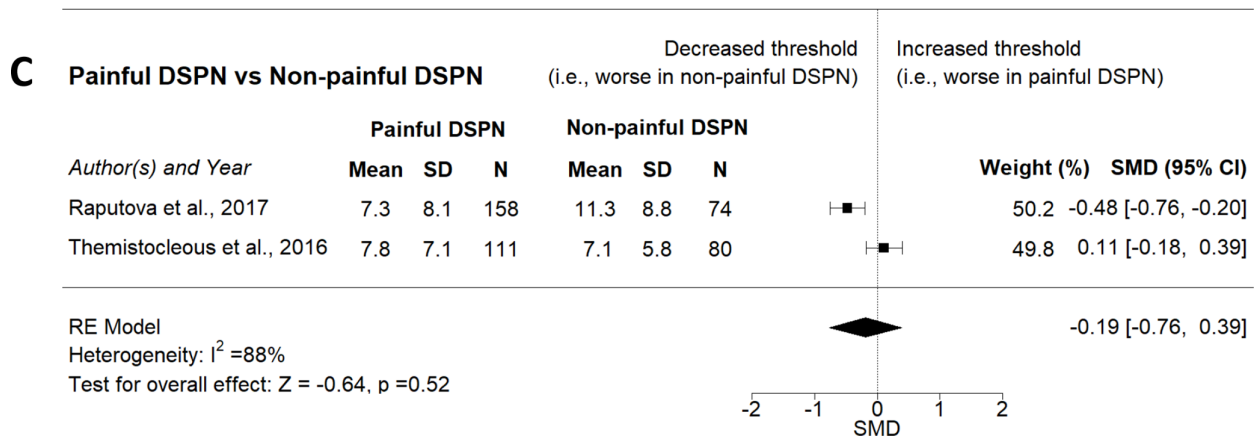

Figure 2 Forest plots for cold pain threshold in patients with painful DSPN versus DSPN, DSPN versus diabetes and diabetes versus healthy. DSPN, distal symmetrical polyneuropathy; RE, random effect; SMD, standardized mean difference.

\section{Cold pain threshold}

Four studies 19363746 with high heterogeneity $\left(\mathrm{I}^{2}=76 \%\right)$ demonstrated that patients with diabetes without DSPN $(n=118)$ had increased cold threshold (ie, pain at lower temperatures) compared with healthy individuals $(\mathrm{n}=165)$ (SMD: $-0.71,95 \%$ CI -1.25 to $0.17 ; \mathrm{Z}=-2.59, \mathrm{p}=0.01$ ). Two studies $^{14} 31$ demonstrated no differences in cold pain threshold between patients with painful DSPN (n=269) and patients with non-painful DSPN (n=154) (SMD: -0.19 , $95 \%$ CI -0.76 to $0.39 ; \mathrm{Z}=-0.64, \mathrm{p}=0.52)$. Three studies ${ }^{19} 2946$ with moderate heterogeneity $\left(\mathrm{I}^{2}=59 \%\right)$ showed that patients with non-painful DSPN $(n=159)$ had increased cold threshold compared with patients with diabetes without DSPN ( $\mathrm{n}=252$ ) (SMD: $-0.76,95 \%$ CI -1.20 to 0.33 ; $\mathrm{Z}=-3.45$, $\mathrm{p}<0.001$ ) (figure 2).

\section{Pressure pain threshold}

Two studies ${ }^{14} 31$ with low heterogeneity $\left(I^{2}=8 \%\right)$ that compared patients with painful DSPN $(n=269)$ and patients with non-painful DSPN $(n=144)$ showed no difference in pressure pain threshold between groups
(SMD 0.14, 95\% CI -0.07 to $0.36 ; \mathrm{Z}=1.33, \mathrm{p}=0.18$ ) (figure 3). Three studies ${ }^{1626} 27$ with moderate heterogeneity $\left(\mathrm{I}^{2}=73 \%\right)$ revealed no differences in pressure pain threshold between patients with non-painful DSPN $(\mathrm{n}=42)$ and healthy participants $(\mathrm{n}=70)$ (SMD 0.60 , $95 \% \mathrm{CI}-0.20$ to $1.40 ; \mathrm{Z}=1.46, \mathrm{p}=0.14$ ) (online supplementary figure 3 ). Findings from one study ${ }^{32}$ could not be pooled because the authors used a cut-off point (seven on a Visual Analogue Scale) rather than the amount of pressure that coincided with the first experience of painful pressure.

\section{Pain threshold by means of electrical stimulation}

Two studies ${ }^{33} 34$ with high heterogeneity $\left(I^{2}=80 \%\right)$ showed that patients with diabetes without DSPN $(n=70)$ had an increased pain threshold by means of electrical threshold (ie, pain at higher intensity) compared with healthy participants $(\mathrm{n}=50)$ (SMD: 1.26 ; 95\% CI: 0.27 , 2.25; $\mathrm{Z}=2.49, \mathrm{p}=0.01$ ) (figure 4 ). The same studies ${ }^{33} 34$ revealed an increased threshold in patients with nonpainful DSPN $(n=29)$ compared with patients with 
Pressure pain threshold

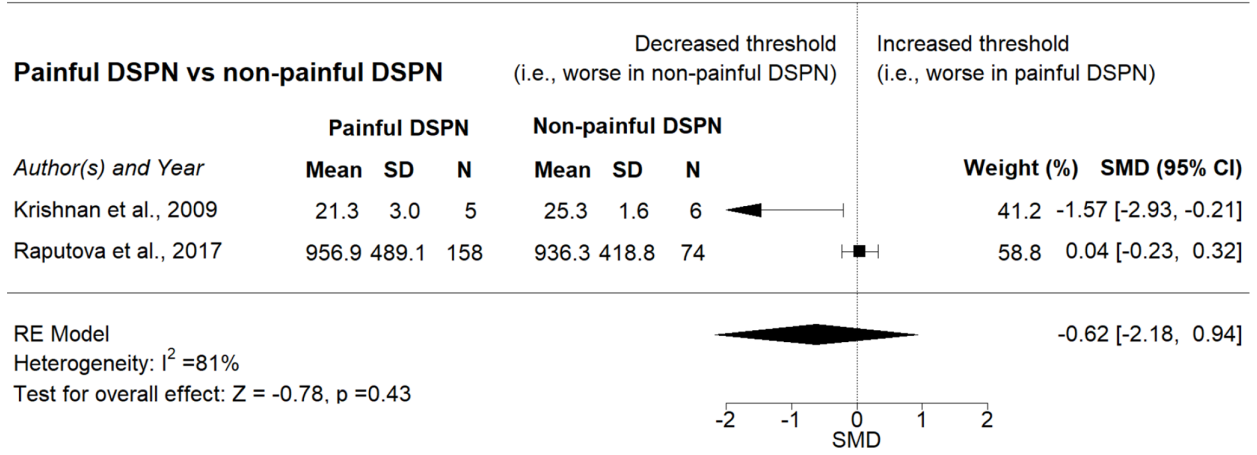

Figure 3 Forest plot for pressure pain threshold in patients with painful DSPN versus non-painful DSPN. DSPN, distal symmetrical polyneuropathy; RE, random effect; SMD, standardized mean difference.

diabetes without DSPN ( $\mathrm{n}=70)$ (SMD: 0.55; 95\% CI: 0.05, $1.05 ; \mathrm{Z}=2.15, \mathrm{p}=0.03)$. Heterogeneity was low for this comparison $\left(I^{2}=13 \%\right)$. These studies ${ }^{33} 34$ with low heterogeneity $\left(\mathrm{I}^{2}=0 \%\right)$ revealed that patients with non-painful DSPN $(n=29)$ had an increased threshold compared with healthy individuals $(\mathrm{n}=50)$ (SMD: $1.85,95 \%$ CI 1.30 to $2.39 ; \mathrm{Z}=6.63, \mathrm{p}<0.001$ ) (online supplementary figure 4 ).

\section{Contact heat-evoked potentials}

Two studies ${ }^{1735}$ with high heterogeneity $\left(\mathrm{I}^{2}=78 \%\right)$ demonstrated no differences in contact heat-evoked potentials between patients with non-painful DSPN $(\mathrm{n}=36)$ and healthy individuals $(\mathrm{n}=42)$ (SMD: $-0.55,95 \% \mathrm{CI}-1.81$ to $0.70 ; \mathrm{Z}=-0.87, \mathrm{p}=0.39$ ) (online supplementary figure 5 ).

\section{Pain modulation}

Pain modulation mechanisms could not be pooled. One study ${ }^{38}$ showed that patients with a longer duration $(>2$ years) of painful DSPN ( $n=20)$ had more efficient conditioned pain modulation compared with patients with shorter duration ( $\leq 2$ years) of painful DSPN $(n=13)$ and compared with healthy individuals $(\mathrm{n}=29)$. Moreover, a less pronounced temporal summation was observed in patients with longer painful DSPN duration compared with shorter painful DSPN duration. No differences were shown in any pain modulation variable between patients with longer painful DSPN duration and healthy individuals. Another study ${ }^{32}$ reported no differences in temporal

Pain threshold by means of electrical stimulation

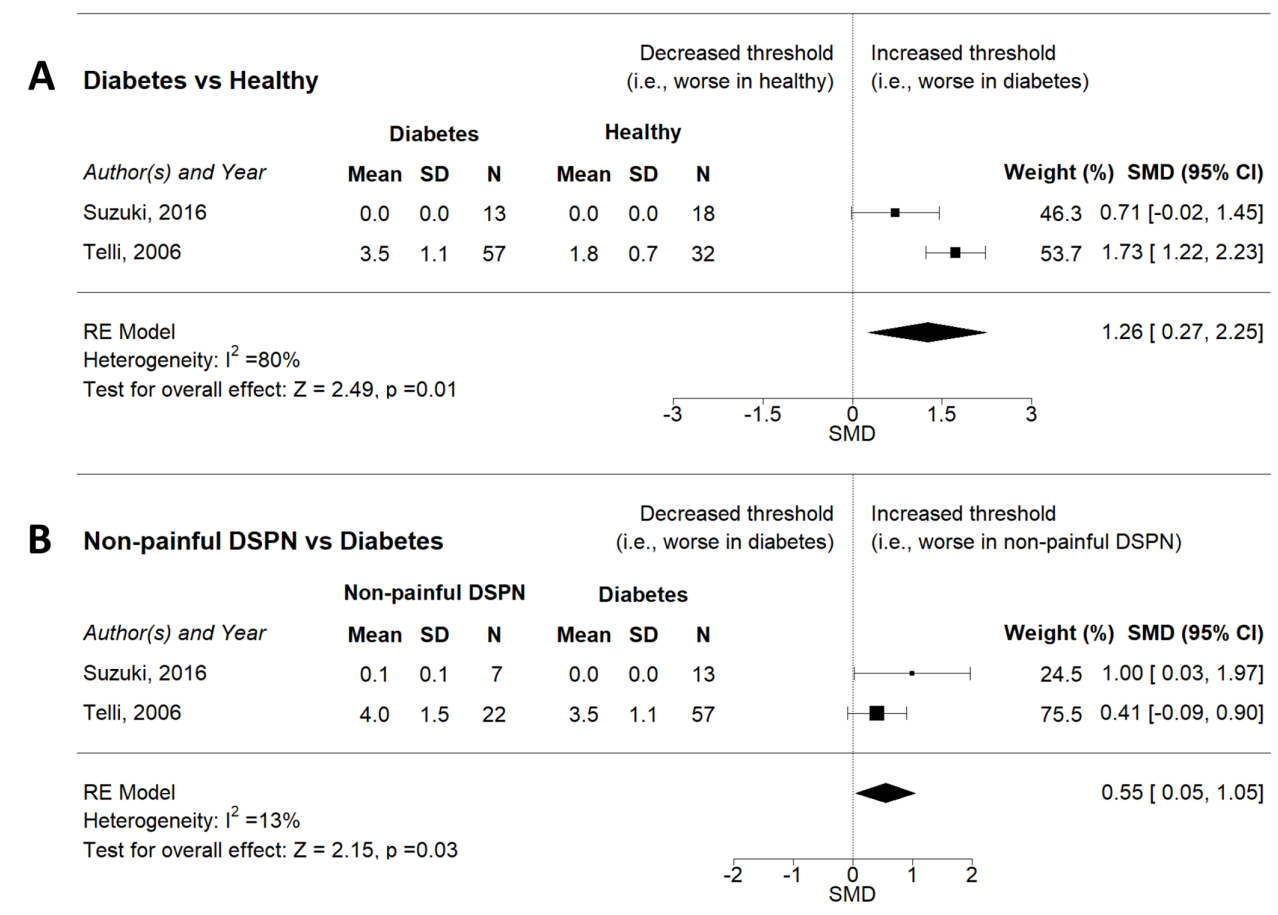

Figure 4 Forest plots for pain threshold by means of electrical stimulation in patients with non-painful DSPN versus diabetes and diabetes versus healthy. DSPN, distal symmetrical polyneuropathy; RE, random effect; SMD, standardized mean difference. 
summation between patients with non-painful DSPN and healthy individuals.

\section{Metaregression analysis}

Due to the small number of studies included in the meta-analyses $(<10$ studies per outcome), we could not perform a meta-regression analysis. ${ }^{49}$

\section{CONCLUSIONS}

This systematic review and meta-analysis revealed four important findings: (1) patients with diabetes without symptoms of DSPN already show loss of function of smalldiameter nerve fibers; (2) loss of small-diameter nerve fiber function is progressive across the diabetes groups (diabetes without DSPN vs diabetes with non-painful DSPN; diabetes with non-painful DSPN vs diabetes with painful DSPN); (3) there is no overall gain of function of small-dimeter nerve fibers in patients with painful DSPN; and (4) central nervous system pain modulation mechanisms are understudied in diabetes with or without DSPN.

This review demonstrated that loss of small-diameter nerve fiber function already occurs in patients with diabetes without symptoms of DSPN. In clinical settings, DSPN is typically diagnosed based on patient-reported symptoms. Therefore, early signs of DSPN may be overlooked unless a specific evaluation of the somatosensory pathways is performed. Routine electrodiagnostic test methods (conduction velocity) are insufficient as they assess predominantly large-diameter nerve fiber function. If increased sensory thresholds indicate a preclinical state of DSPN, QST or bedside neurological testing, including small-diameter nerve fiber function, could potentially be a valuable non-invasive tool to assist in early diagnosis, and possibly prevention and management of DSPN. Furthermore, the fact that small-diameter nerve fiber neuropathy is already establishing before symptoms of DSPN become apparent may be an important realization for people with diabetes. It may be a convincing educational message for patients with diabetes to adhere to healthy lifestyle guidelines. ${ }^{50} \mathrm{C}$-fibers play a crucial role in tissue healing, and dysfunction in these unmyelinated small-diameter nerve fibers may increase the morbidity of cutaneous and subcutaneous conditions due to the delayed healing, and risk of infections, even in people with diabetes without symptoms of DSPN. ${ }^{51}$

The greater loss of small-diameter nerve fiber function for patients with non-painful DSPN compared with patients without symptoms of DSPN is in line with expectations. Loss of small-diameter nerve fiber function is commonly observed in other polyneuropathies, such as in people with HIV-associated peripheral neuropathy ${ }^{52}$ or Guillain-Barre syndrome. ${ }^{53}$

For painful neuropathies, hyperalgesia and allodynia are typically associated with gain of small-diameter nerve fiber function and/or altered postsynaptic processing in spinal and supra-spinal neurons. ${ }^{54}$ People with mononeuropathies (eg, radiculopathy ${ }^{55}$ ) and polyneuropathies (eg, chemotherapy-induced neuropathy ${ }^{56}$ ) show a gain of small-diameter nerve fiber function for various QST modalities. The observed greater loss of small-diameter nerve function in people with painful DSPN compared with people with non-painful DSPN is therefore paradoxical. Painful DSPN cannot be explained by static QST measures.

Other methods, such as corneal confocal microscopy ${ }^{57}$ and MRI ${ }^{58}$ have identified differences between people with painful DSPN and non-painful DSPN. Imaging studies using MRI have shown that people with painful DSPN characterized by loss of function have a reduction in spinal cord and primary somatosensory cortical grey matter volume compared with patients with non-painful DSPN ${ }^{58}$ Furthermore, patients with painful-DSPN appear to have greater thalamic vascularity at rest with increased relative cerebral blood flow compared with patients with no DSPN and painless DSPN. ${ }^{59}$ It has been suggested that different processing of nociceptive signals occurs in the central nervous system's pain modulatory system, resulting in reduced inhibition and increased amplification in patients with painful DSPN. ${ }^{60}$ This can be evaluated with dynamic QST measures (such as conditioned pain modulation and temporal and special summation). Unfortunately, our review revealed that these measures are understudied in diabetes.

An important consideration is that in the original studies, and therefore also in the current review and metaanalysis, the results are reported as either loss or gain of nerve function at group level. Rather than analyzing QST modalities separately, there is a recent evolution to various group QST modalities together to create sensory phenotypes. Large phenotyping studies ${ }^{14} 3161$ revealed that although loss of nerve function is the most prevalent phenotype $(64 \%)$ in patients with painful DSPN, a substantial portion of patients have a gain of function phenotype (thermal hyperalgesia: 13\%, mechanical hyperalgesia: $19 \%$ (4\% had a profile similarly to healthy participants) ). ${ }^{61}$ The phenotype loss of function and the phenotype gain of function are also both present in other neuropathies, such as postherpetic neuralgia. ${ }^{62}$ This illustrates that although we did observe loss of nerve function at group level (diabetes without symptoms of DSPN, patients with non-painful DSPN and patients with painful DSPN), individual assessment of small-diameter nerve fiber function remains important.

A limitation of the review is that most comparisons showed moderate or high heterogeneity. It is important to note that most of the studies included a mix of patients with type 1 and type 2 diabetes. Although the mechanisms underlying diabetic neuropathy remain not fully understood ${ }^{63}$ it is apparent that different mechanisms are present in diabetic neuropathy in patients with type 1 and type 2 diabetes. ${ }^{6465}$ The source of heterogeneity between studies could be influenced by differences in sample size, sampling methods, and population characteristics, including the composition of the sample (type 1 
and/or type 2 diabetes), diversity of QST protocols ${ }^{66}$ and criteria to diagnose DSPN. The lack of reporting population characteristics and small sample sizes may also influence heterogeneity, as pain thresholds are known to vary by sex ${ }^{67}$ or age.$^{68}$ Furthermore, consistent with a previous systematic review on QST ${ }^{66}$ we found large variability in the reporting of QST protocols. We recommend that researchers and clinicians adhere to published guidelines on the use and reporting of $\mathrm{QST}^{69}$ and to report the results for patients with type 1 and type 2 diabetes separately.

\section{Author affiliations}

${ }^{1}$ School of Allied Health Sciences, Griffith University, Gold Coast, Queensland, Australia

${ }^{2}$ Faculty of Human Movement Sciences, Free University Amsterdam, Amsterdam, The Netherlands

${ }^{3}$ Menzies Health Institute Queensland, Griffith University, Nathan, Queensland, Australia

${ }^{4}$ Amsterdam Movement Sciences, Vrije Universiteit Amsterdam, Amsterdam, The Netherlands

Contributors ES-S, MWC and LB developed the idea for the review. ES-S developed the search strategy, selection criteria and data extraction form, with guidance from MWC and LB. ES-S and MS performed the literature searches, screened the literature, extracted the data and appraised the quality of the included papers. LB acted as third reviewer when necessary. ES-S developed the meta-analysis plan and designed the figures and tables. ES-S and MWC wrote the manuscript, with input from LB. All authors read and approved the final version of the manuscript.

Funding The authors have not declared a specific grant for this research from any funding agency in the public, commercial or not-for-profit sectors.

Competing interests None declared.

Patient consent for publication Not required.

Ethics approval This systematic review and meta-analysis was based on published data. As researchers did not access any information that could lead to the identification of an individual patient, no concerning ethical issue was raised in this research. Therefore, obtaining ethical approval and consent of participants was waived.

Provenance and peer review Not commissioned; externally peer reviewed.

Data availability statement Data are available upon reasonable request. The datasets generated during and/or analyzed during the current study are available from the corresponding author on reasonable request.

Open access This is an open access article distributed in accordance with the Creative Commons Attribution Non Commercial (CC BY-NC 4.0) license, which permits others to distribute, remix, adapt, build upon this work non-commercially, and license their derivative works on different terms, provided the original work is properly cited, appropriate credit is given, any changes made indicated, and the use is non-commercial. See: http://creativecommons.org/licenses/by-nc/4.0/.

ORCID ID

Michel W Coppieters http://orcid.org/0000-0003-4534-8564

\section{REFERENCES}

1 World Health Organization. Global report on diabetes. Geneva; 2016.

2 Tesfaye S. Recent advances in the management of diabetic distal symmetrical polyneuropathy. J Diabetes Investig 2011;2:33-42.

3 Truini A, Spallone V, Morganti R, et al. A cross-sectional study investigating frequency and features of definitely diagnosed diabetic painful polyneuropathy. Pain 2018;159:2658-66.

4 Dyck PJ, Albers JW, Andersen H, et al. Diabetic polyneuropathies: update on research definition, diagnostic criteria and estimation of severity. Diabetes Metab Res Rev 2011;27:620-8.

5 Pop-Busui R, Boulton AJM, Feldman EL, et al. Diabetic neuropathy: a position statement by the American diabetes association. Diabetes Care 2017;40:136-54.
6 Tesfaye S, Boulton AJM, Dyck PJ, et al. Diabetic neuropathies: update on definitions, diagnostic criteria, estimation of severity, and treatments. Diabetes Care 2010;33:2285-93.

7 Hovaguimian A, Gibbons CH. Diagnosis and treatment of pain in small-fiber neuropathy. Curr Pain Headache Rep 2011;15:193-200.

8 Andersen H. Motor dysfunction in diabetes. Diabetes Metab Res Rev 2012;28 Suppl 1:89-92.

9 Tesfaye S, Chaturvedi N, Eaton SEM, et al. Vascular risk factors and diabetic neuropathy. N Engl J Med 2005;352:341-50.

10 Boulton AJM, Vileikyte L, Ragnarson-Tennvall G, et al. The global burden of diabetic foot disease. Lancet 2005;366:1719-24.

11 Clarke C, Christensen C, Curran MWT, et al. Assessment of small sensory fiber function across the spectrum of severity in carpal tunnel syndrome patients. Muscle Nerve 2017:56:814-6.

12 Morellini N, Finch PM, Goebel A, et al. Dermal nerve fibre and mast cell density, and proximity of mast cells to nerve fibres in the skin of patients with complex regional pain syndrome. Pain 2018;159:2021-9.

13 Huang ES, Laiteerapong N, Liu JY, et al. Rates of complications and mortality in older patients with diabetes mellitus: the diabetes and aging study. JAMA Intern Med 2014;174:251-8.

14 Raputova J, Srotova I, Vlckova E, et al. Sensory phenotype and risk factors for painful diabetic neuropathy: a cross-sectional observational study. Pain 2017;158:2340-53.

15 Themistocleous AC, Ramirez JD, Shillo PR, et al. The pain in neuropathy study (pins). Pain 2016;157:1132-45.

16 Wienemann T, Chantelau EA, Richter A. Pressure pain perception at the injured foot: the impact of diabetic neuropathy. J Musculoskelet Neuronal Interact 2012;12:254-61.

17 Parson HK, Nguyen VT, Orciga M-A, et al. Contact heat-evoked potential stimulation for the evaluation of small nerve fiber function. Diabetes Technol Ther 2013;15:150-7.

18 Ragé M, Van Acker N, Knaapen MWM, et al. Asymptomatic small fiber neuropathy in diabetes mellitus: investigations with intraepidermal nerve fiber density, quantitative sensory testing and laser-evoked potentials. J Neurol 2011;258:1852-64.

19 Yildiz N, Dogan N, Yildiz S, et al. The evaluation of small nerve fiber dysfunction with quantitative sensory testing in patients with type II diabetes mellitus without large fiber neuropathy, and normal values for thermal thresholds. Noropsikiyatri Ars 2010.

20 Moher D, Liberati A, Tetzlaff J, et al. Preferred reporting items for systematic reviews and meta-analyses: the PRISMA statement. BMJ 2009;339:b2535.

21 Sierra-Silvestre E, Bisset L, Coppieters MW. Altered pain processing in people with type I and II diabetes: a protocol for a systematic review and meta-analysis of pain threshold and pain modulation mechanisms. Syst Rev 2018;7:222.

22 Veritas Health Innovation, Melbourne A. Covidence systematic review sofware. Covidence 2016.

23 Higgins J, Green S. Cochrane Handbook for systematic reviews of interventions. Oxford, UK: Cochrane Collab, 2011. http://handbook. cochrane.org

24 Downs SH, Black N. The feasibility of creating a checklist for the assessment of the methodological quality both of randomised and non-randomised studies of health care interventions. J Epidemiol Community Health 1998;52:377-84.

25 Viechtbauer W. Conducting Meta-Analyses in $R$ with the metafor Package. J Stat Softw 2010;36:1-48.

26 Chantelau EA. Conventional deep pressure algometry is not suitable for clinical assessment of nociception in painless diabetic neuropathy. Diabet Foot Ankle 2016;7:31922.

27 Chantelau E, Wienemann T, Richter A. Pressure pain thresholds at the diabetic charcot-foot: an exploratory study. J Musculoskelet Neuronal Interact 2012.

28 Claus D, Hilz MJ, Hummer I, et al. Methods of measurement of thermal thresholds. Acta Neurol Scand 1987;76:288-96.

29 Guo W, Li Y-M, Ai Z-H, et al. [Joint diagnostic value of four temperature sensation tests in elderly patients with type 2 diabetic peripheral neuropathy]. Sichuan Da Xue Xue Bao Yi Xue Ban 2013:44:673-6.

30 Quattrini C, Tavakoli M, Jeziorska M, et al. Surrogate markers of small fiber damage in human diabetic neuropathy. Diabetes 2007;56:2148-54.

31 Themistocleous AC, Ramirez JD, Shillo PR, et al. The pain in neuropathy study (pins): a cross-sectional observational study determining the somatosensory phenotype of painful and painless diabetic neuropathy. Pain 2016;157:1132-45.

32 Søfteland E, Brock C, Frøkjær JB, et al. Association between visceral, cardiac and sensorimotor polyneuropathies in diabetes mellitus. J Diabetes Complications 2014;28:370-7. 
33 Suzuki C, Kon T, Funamizu Y, et al. Elevated pain threshold in patients with asymptomatic diabetic neuropathy: an intraepidermal electrical stimulation study. Muscle Nerve 2016;54:146-9.

34 Telli O, Cavlak U. Measuring the pain threshold and tolerance using electrical stimulation in patients with type II diabetes mellitus. $J$ Diabetes Complications 2006;20:308-16.

35 Wong M-C, Chung JWY. Feasibility of contact heat evoked potentials for detection of diabetic neuropathy. Muscle Nerve 2011;44:902-6.

36 Yin H-min, Feng W, Ding M-ping. [The significance of quantitative temperature sense thresholds in diagnosis of small fibrous sensory neuropathy in patients with type 2 diabetes]. Zhongguo Ying Yong Sheng Li Xue Za Zhi 2015;31:150-3.

37 Ziegler D, Mayer P, Wiefels $\mathrm{K}$, et al. Assessment of small and large fiber function in long-term type 1 (insulin-dependent) diabetic patients with and without painful neuropathy. Pain 1988;34:1-10.

38 Granovsky $Y$, Nahman-Averbuch $\mathrm{H}$, Khamaisi M, et al. Efficient conditioned pain modulation despite pain persistence in painful diabetic neuropathy. Pain Rep 2017;2:e592.

39 Diemel LT, Cai F, Anand P, et al. Increased nerve growth factor mRNA in lateral calf skin biopsies from diabetic patients. Diabet Med 1999;16:113-9.

40 Pittenger GL, Ray M, Burcus NI, et al. Intraepidermal nerve fibers are indicators of small-fiber neuropathy in both diabetic and nondiabetic patients. Diabetes Care 2004;27:1974-9.

41 Krishnan STM, Quattrini C, Jeziorska M, et al. Abnormal LDIflare but normal quantitative sensory testing and dermal nerve fiber density in patients with painful diabetic neuropathy. Diabetes Care 2009;32:451-5.

42 Chao C-C, Tseng M-T, Lin Y-J, et al. Pathophysiology of neuropathic pain in type 2 diabetes: skin denervation and contact heat-evoked potentials. Diabetes Care 2010;33:2654:1974-2659.

43 Krämer HH, Rolke R, Bickel A, et al. Thermal thresholds predict painfulness of diabetic neuropathies. Diabetes Care 2004;27:2386-91.

44 Krishnan STM, Baker NR, Carrington AL, et al. Comparative roles of microvascular and nerve function in foot ulceration in type 2 diabetes. Diabetes Care 2004;27:1343-8.

45 Levy D, Abraham R, Reid G. A comparison of two methods for measuring thermal thresholds in diabetic neuropathy. J Neurol Neurosurg Psychiatry 1989;52:1072-7.

46 Petropoulos IN, Ferdousi M, Marshall A, et al. The inferior Whorl for detecting diabetic peripheral neuropathy using corneal confocal microscopy. Invest Ophthalmol Vis Sci 2015;56:2498.

47 Redmond JM, McKenna MJ, Feingold M, et al. Sensory testing versus nerve conduction velocity in diabetic polyneuropathy. Muscle Nerve 1992;15:1334-9.

48 Dyck PJ. Detection, characterization, and staging of polyneuropathy: assessed in diabetics. Muscle Nerve 1988;11:21-32.

49 Higgins JP, Green S. Cochrane Handbook for systematic reviews of interventions, 2008.

50 Chatterjee S, Davies MJ, Heller S, et al. Diabetes structured selfmanagement education programmes: a narrative review and current innovations. Lancet Diabetes Endocrinol 2018;6:130-42.
51 Barsun A, Sen S, Palmieri TL, et al. A ten-year review of lower extremity burns in diabetics: small burns that lead to major problems. J Burn Care Res 2013;34:255-60.

52 Phillips TJC, Brown M, Ramirez JD, et al. Sensory, psychological, and metabolic dysfunction in HIV-associated peripheral neuropathy: a cross-sectional deep profiling study. Pain 2014;155:1846-60.

53 Martinez V, Fletcher D, Martin F, et al. Small fibre impairment predicts neuropathic pain in Guillain-Barré syndrome. Pain 2010;151:53-60.

54 Woolf CJ. Central sensitization: implications for the diagnosis and treatment of pain. Pain 2011;152:S2-15.

55 Tampin B, Slater H, Hall T, et al. Quantitative sensory testing somatosensory profiles in patients with cervical radiculopathy are distinct from those in patients with nonspecific neck-arm pain. Pain 2012;153:2403-14

56 Geber C, Breimhorst M, Burbach B, et al. Pain in chemotherapyinduced neuropathy--more than neuropathic? Pain 2013;154:2877-87.

57 Kalteniece A, Ferdousi M, Azmi S, et al. Keratocyte density is reduced and related to corneal nerve damage in diabetic neuropathy. Invest Ophthalmol Vis Sci 2018;59:3584-90.

58 Selvarajah D, Wilkinson ID, Fang F, et al. Structural and functional abnormalities of the primary somatosensory cortex in diabetic peripheral neuropathy: a multimodal MRI study. Diabetes 2019;68:796-806.

59 Selvarajah D, Wilkinson ID, Gandhi R, et al. Microvascular perfusion abnormalities of the thalamus in painful but not painless diabetic polyneuropathy. Diabetes Care 2011;34:718-20.

60 Shillo P, Sloan G, Greig M, et al. Painful and painless diabetic neuropathies: what is the difference? Curr Diab Rep 2019;19:32.

61 Vollert J, Maier C, Attal N, et al. Stratifying patients with peripheral neuropathic pain based on sensory profiles: algorithm and sample size recommendations. Pain 2017;158:1446-55.

62 Baron R, Maier C, Attal N, et al. Peripheral neuropathic pain: a mechanism-related organizing principle based on sensory profiles. Pain 2017;158:261-72.

63 Feldman EL, Callaghan BC, Pop-Busui R, et al. Diabetic neuropathy. Nat Rev Dis Primers 2019;5:41.

64 Callaghan BC, Little AA, Feldman EL, et al. Enhanced glucose control for preventing and treating diabetic neuropathy. Cochrane Database Syst Rev 2012;6:CD007543.

65 Jende JME, Groener JB, Oikonomou D, et al. Diabetic neuropathy differs between type 1 and type 2 diabetes: insights from magnetic resonance neurography. Ann Neurol 2018;83:588-98.

66 Hübscher M, Moloney N, Leaver A, et al. Relationship between quantitative sensory testing and pain or disability in people with spinal pain-a systematic review and meta-analysis. Pain 2013;154:1497-504

67 Fillingim RB, King CD, Ribeiro-Dasilva MC, et al. Sex, gender, and pain: a review of recent clinical and experimental findings. $J$ Pain 2009;10:447-85.

68 Lautenbacher S, Kunz M, Strate P, et al. Age effects on pain thresholds, temporal summation and spatial summation of heat and pressure pain. Pain 2005;115:410-8.

69 Rolke R, Baron R, Maier C, et al. Quantitative sensory testing in the German research network on neuropathic pain (DFNS): standardized protocol and reference values. Pain 2006;123:231-43. 